\title{
The association between ECV and microcirculation perfusion abnormalities in non-ischemic dilated cardiomyopathy
}

\author{
Aamir Ali ${ }^{1 *}$, Li-Yueh Hsu², Ankur Gulati ${ }^{1}$, Tevfik Ismail ${ }^{1}$, Claire E Raphael', Vassilis Vassiliou', Navtej Chahal ${ }^{1}$, \\ Kaushiga Krishnathansan', Natasha Davendralingam', Carla Goncalves', Ricardo Wage ${ }^{1}$, Pedro Ferreira', \\ Arun J Baksi ${ }^{1}$, Peter Gatehouse ${ }^{1}$, David Firmin', Dudley J Pennell ${ }^{1}$, Peter Kellman², Andrew E Arai ${ }^{2}$, \\ Sanjay K Prasad
}

From 17th Annual SCMR Scientific Sessions New Orleans, LA, USA. 16-19 January 2014

\section{Background}

Myocardial fibrosis and abnormalities of the microcirculation are features of non-ischemic dilated cardiomyopathy (DCM) and may contribute to adverse remodeling. However, relationship between perfusion abnormalities and diffuse fibrosis has not been fully characterised. CMR allows quantification of the extracellular volume fraction (ECV), a marker of fibrosis, and absolute myocardial blood flow, in a single study. We hypothesised that increased ECV was associated with impaired myocardial perfusion reserve (MPR) in DCM patients.

\section{Methods}

Consecutive DCM patients referred for a clinical CMR study and age/gender-matched controls were prospectively enrolled. All subjects underwent CMR (1.5T Siemens Avanto) according to a standardized protocol which included T1-mapping and first-pass perfusion imaging. Mid-ventricular short-axis T1-parameter maps were acquired using a Modified Look-Locker Inversion recovery sequence prior to contrast and 20 minutes after gadolinium administration (Gadobutrol $0.1 \mathrm{mmol} / \mathrm{kg}$ ). The preand post-contrast T1-maps were co-registered and using the haematocrit, an ECV map generated (Figure 1). CMR first-pass perfusion imaging was performed using a hybrid echo-planar-imaging sequence at the corresponding T1map slice position during adenosine-induced hyperemia $(140 \mu \mathrm{g} / \mathrm{kg} / \mathrm{min})$ and 30 minutes later at rest. Myocardial perfusion reserve (MPR) was calculated from absolute

\footnotetext{
${ }^{1}$ CMR Unit, Royal Brompton Hospital, London, UK

Full list of author information is available at the end of the article
}

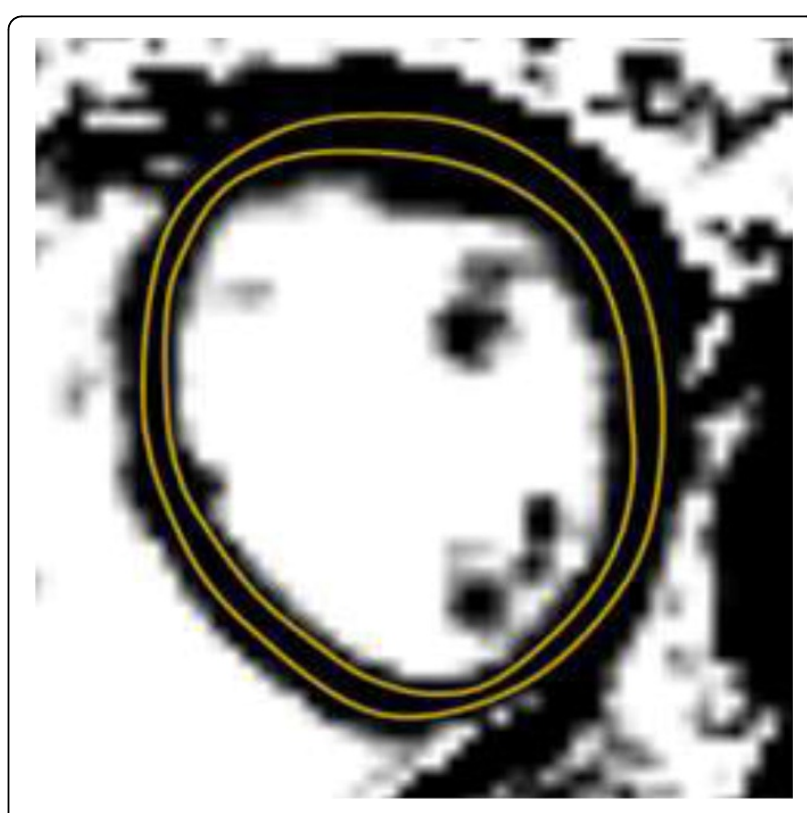

Figure 1 Example of short axis ECV Map with the region of interest placed in a single mid-ventricular slice.

stress and rest global myocardial blood flow quantified by a Fermi-constrained deconvolution algorithm.

\section{Results}

Thirty two DCM patients ( 25 male, mean age $49 \pm 15 \mathrm{yrs,}$ mean left ventricular ejection fraction [LVEF] $38 \pm 11 \%$ ) and 28 controls (17 male, mean age $47 \pm 13$ yrs, mean LVEF $68 \pm 5 \%$ ) were studied. Baseline clinical and CMR data are summarized in Table 1. Patients with DCM had a 
Table 1 Baseline Clinical and CMR Characteristics

\begin{tabular}{cccc}
\hline Characteristic & DCM $(\mathbf{n}=\mathbf{3 2})$ & Control $(\mathbf{n}=\mathbf{2 8})$ & $\boldsymbol{p}$-value \\
\hline Age (years) & 49 & 47 & 0.552 \\
Male $(\mathbf{n})$ & 25 & 17 & 0.167 \\
Heart rate $(\mathbf{b p m})$ & 74 & 62 & 0.001 \\
Systolic BP $(\mathbf{m m H g})$ & 122 & 120 & 0.701 \\
Diastolic BP $(\mathbf{m m H g})$ & 74 & 76 & 0.626 \\
LV-EDVi $\left(\mathbf{m L} / \mathbf{m}^{\mathbf{2}}\right)$ & 149 & 82 & $<0.001$ \\
LV-ESVi $\left(\mathbf{m L} / \mathbf{m}^{\mathbf{2}}\right)$ & 96 & 26 & $<0.001$ \\
LVEF $(\%)$ & 38 & 68 & $<0.001$ \\
LVMI $\left(\mathbf{g} / \mathbf{m}^{\mathbf{2}}\right)$ & 91 & 28 & $<0.001$ \\
\hline
\end{tabular}

Beats per minute (bpm); blood pressure (BP); dilated cardiomyopathy (DCM); indexed left ventricular end-diastolic volume (LV-EDVi); indexed left ventricular end-systolic volume (LV-ESVi); left ventricular ejection fraction (LVEF); indexed left ventricular mass index (LVMI)

significantly higher ECV (mean \pm SD $28.7 \pm 3.6 \%$ vs $25.7 \pm$ 3.4\%, $\mathrm{p}<0.001) .6$ (18\%) DCM patients had an ECV more than 2 SD above the control group. DCM patients had a lower MPR compared to controls $(1.73 \pm 0.63$ vs $2.53 \pm$ $0.81, \mathrm{p}<0.001)$. Linear regression analysis demonstrated a significant but weak association between ECV and MPR $(\mathrm{B}=-1.2,95 \% \mathrm{CI}-2.36$ to $-0.04, \mathrm{R} 2=0.07, \mathrm{p}<0.001)$.

\section{Conclusions}

ECV is raised and MPR is impaired in DCM. There is a significant but weak association between these two parameters. Further work is required to assess if a temporal relationship exists between MPR and ECV, as well whether they individually correlate with markers of disease severity.

\section{Funding}

This project was supported by the NIHR Cardiovascular Biomedical Research Unit of Royal Brompton and Harefield NHS Foundation Trust, the British Heart Foundation, and CORDA (research charity). Dr Andrew Arai, Dr Peter Kellman and Dr Li-Yueh Hsu are funded by the National Heart, Lung and Blood Institute, NIH, Bethesda, MD, USA.

\section{Authors' details}

${ }^{1}$ CMR Unit, Royal Brompton Hospital, London, UK. ${ }^{2}$ National Institutes of Health, Bethesda, Maryland, USA.

Published: 16 January 2014

doi:10.1186/1532-429X-16-S1-088

Cite this article as: Ali et al:: The association between ECV and microcirculation perfusion abnormalities in non-ischemic dilated cardiomyopathy. Journal of Cardiovascular Magnetic Resonance 201416 (Suppl 1):O88.
Submit your next manuscript to BioMed Central and take full advantage of:

- Convenient online submission

- Thorough peer review

- No space constraints or color figure charges

- Immediate publication on acceptance

- Inclusion in PubMed, CAS, Scopus and Google Scholar

- Research which is freely available for redistribution

Submit your manuscript at www.biomedcentral.com/submit 